\title{
Microtubule Disorganization Affects the Mitochondrial Permeability Transition Pore in Cardiac Myocytes
}

\author{
Azumi Kumazawa, MD; Hideki Katoh, MD; Daishi Nonaka, MD; Tomoyuki Watanabe, MD; \\ Masao Saotome, MD; Tsuyoshi Urushida, MD; Hiroshi Satoh, MD; Hideharu Hayashi, MD
}

\begin{abstract}
Background: Microtubule (MT) disorganization is related to cardiac disorders. To elucidate the mechanism by which disorganization of the MT network deteriorates cardiac function, the relationship between MT disorganization and mitochondrial permeability transition pore (MPTP) in cardiac myocytes was investigated.
\end{abstract}

\begin{abstract}
Methods and Results: The effects of MT stabilization (by paclitaxel) and MT disruption (by nocodazole) on mitochondrial membrane potential $\left(\Delta \psi_{\mathrm{m}}\right)$ and the opening of $\mathrm{mPTP}$ were measured in permeabilized Sprague-Dawley rat myocytes. Both paclitaxel and nocodazole depolarized $\Delta \psi_{\mathrm{m}}$ and opened $\mathrm{mPTP}$. When isolated mitochondria were exposed to paclitaxel or nocodazole, there were no changes in $\Delta \psi_{\mathrm{m}}$. The effects of paclitaxel or nocodazole on $\Delta \psi_{\mathrm{m}}$ depolarization and mPTP were inhibited by cyclosporin A. Treatment of myocytes with 0Ca+BAPTA or inhibition of sarcoplasmic reticulum (SR) $\mathrm{Ca}^{2+}$ uptake by thapsigargin prevented the effect of paclitaxel on $\mathrm{mPTP}$, but not that of nocodazole. Inhibition of the mitochondrial $\mathrm{Ca}^{2+}$ uniporter by $\mathrm{Ru} 360$ did not alter the effect of paclitaxel on mPTP. Paclitaxel reduced the expression of the mitochondrial fusion protein, mitofusin-2, and induced mitochondrial fragmentation.
\end{abstract}

Conclusions: Disruption of the MT network by nocodazole might destroy the MT-mitochondria connection and alter mitochondrial function. MT disorganization by paclitaxel could regulate MPTP through the outer mitochondrial membrane complex and the $\mathrm{Ca}^{2+-}$-sensitive signaling pathway, which also interacts with the mitochondrial fusion protein, mitofusin-2. (Circ J 2014; 78: 1206-1215)

Key Words: Calcium ions; Microtubules; Mitochondrial membrane potential; Mitochondrial permeability transition pore; Mitofusin-2

$\mathbf{M}$ icrotubules (MTs) are a major component of the cytoskeleton, and play critical roles in multiple cellular processes such as mitosis, intracellular transport and cell morphogenesis. MTs exist in equilibrium between polymerized and depolymerized states of $\alpha$ - and $\beta$-tubulin heterodimer, and an unbalance of this equilibrium causes cellular and organic dysfunction. ${ }^{1}$

In the heart, disorganization of the MT network has been implicated in the progression of heart disease. MT content was increased in pressure overload cardiac hypertrophy or in patients with severe aortic stenosis. ${ }^{2,3}$ MTs were reversibly disrupted by reperfusion after a brief period of ischemia and this contributes to the development of stunned myocardium. ${ }^{4}$

Mitochondria regulate cell fate by promoting apoptosis and necrosis. The transient or sustained opening of the mitochondrial permeability transition pore (mPTP), a multiple-protein complex formed at the contact site between the inner and the outer mitochondrial membrane (IMM and OMM, respectively), plays key roles in apoptosis and necrosis, and mitochondrial
$\mathrm{Ca}^{2+}$ concentration $\left(\left[\mathrm{Ca}^{2+}\right]_{\mathrm{m}}\right)$ is a strong inducer of $\mathrm{mPTP}$ opening. 5

Mitochondria are mobile organelles and move along with MTs in many cell types. ${ }^{6}$ In neuronal cells, disorganization of the MT network promotes the opening of mPTP. ${ }^{7}$ The MT stabilizer, paclitaxel, induces mPTP and alters cytosolic $\mathrm{Ca}^{2+}$ concentration, resulting in the promotion of apoptosis. ${ }^{8}$ These findings imply that changes in the MT network could affect both mitochondrial function and $\mathrm{Ca}^{2+}$ homeostasis. In cardiac myocytes, disruption of MTs enhanced $\mathrm{Ca}^{2+}$ signaling. ${ }^{9} \mathrm{How}-$ ever, how the modulation of MTs affects MPTP and cell damage has not been completely explored.

Between mitochondria and MTs, there is a structural complex composed of the cargo protein, kinesin, mitochondrial Rho GTPase (Miro) and connecting protein (Milton). Milton binds Miro and the kinesin heavy chain (KHC), which makes a Miro-Milton-KHC complex. ${ }^{10}$ This complex is important not only for mitochondrial movement, but also for the regulation of mitochondrial function. ${ }^{11}$

Received October 18, 2013; revised manuscript received December 26, 2013; accepted January 14, 2014; released online March 10, 2014 Time for primary review: 19 days

Division of Cardiology, Internal Medicine III, Hamamatsu University School of Medicine, Hamamatsu, Japan

Mailing address: Hideki Katoh, MD, Division of Cardiology, Internal Medicine III, Hamamatsu University School of Medicine, 1-20-1

Handayama, Higashi-ku, Hamamatsu 431-3192, Japan. E-mail: hkatoh@hama-med.ac.jp

ISSN-1346-9843 doi:10.1253/circj.CJ-13-1298

All rights are reserved to the Japanese Circulation Society. For permissions, please e-mail: cj@j-circ.or.jp 


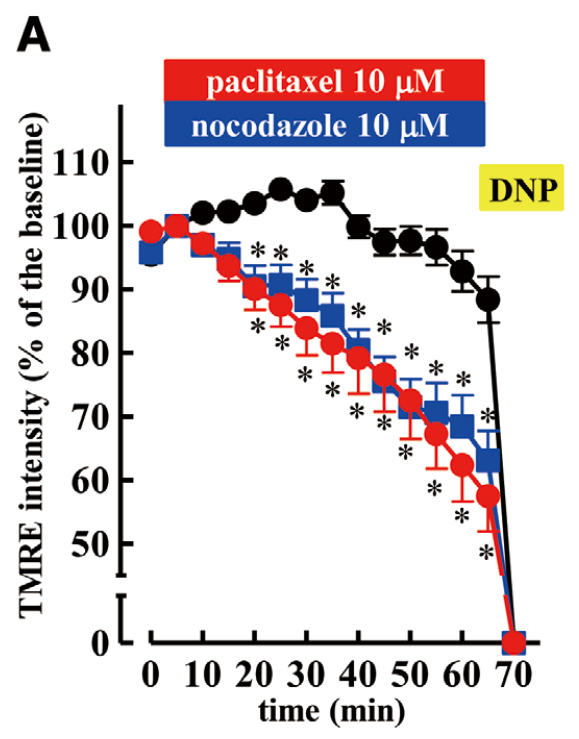

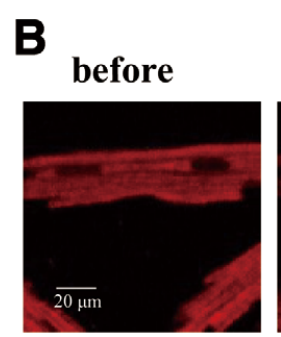
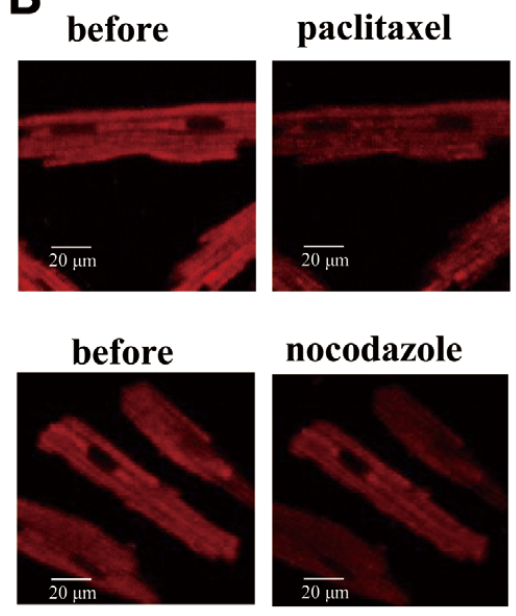

\section{nocodazole}

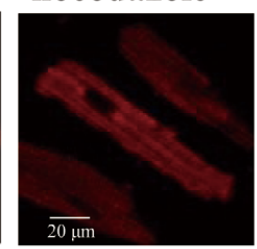

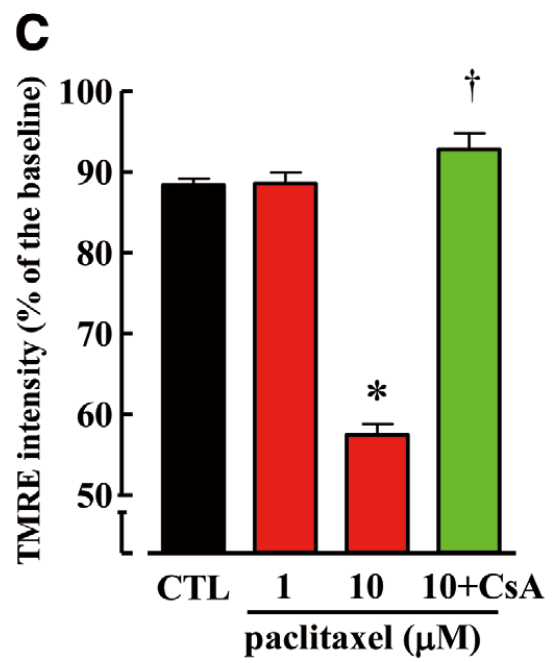

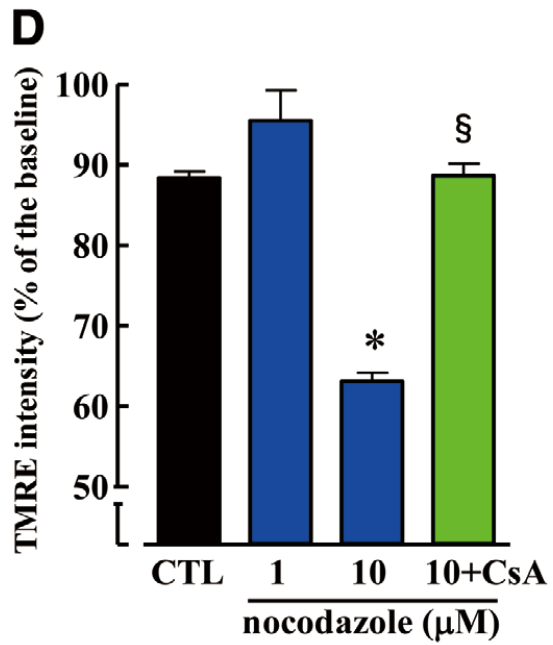

Figure 1. Paclitaxel and nocodazole depolarized the mitochondrial membrane potential. (A) Time-courses of the changes in tetramethylrhodamine ethyl ester (TMRE) intensity. Permeabilized myocytes were perfused with an internal solution $(\mathbf{\bullet} ; n=21)$, and then paclitaxel $(0 ; 10 \mu \mathrm{mol} / \mathrm{L}, \mathrm{n}=17)$ or nocodazole $(\square ; 10 \mu \mathrm{mol} / \mathrm{L}, \mathrm{n}=20)$ was applied. As a reference, an uncoupler, DNP $(100 \mu \mathrm{mol} / \mathrm{L})$, was applied at the end of the experiments. (B) The 2-D images of TMRE - Upper left: before paclitaxel; Upper right: after 60 min perfusion of paclitaxel; Lower left: before nocodazole; and Lower right: after 60 min perfusion of nocodazole. (C) TMRE intensity after 60 min perfusion with an internal solution (CTL, $n=21)$, paclitaxel $(1 \mu \mathrm{mol} / \mathrm{L} ; \mathrm{n}=7,10 \mu \mathrm{mol} / \mathrm{L} ; \mathrm{n}=17)$ and paclitaxel plus cyclosporine $A(C s A ; 0.4 \mu \mathrm{mol} / \mathrm{L}, \mathrm{n}=5)$. In the CsA-treated group, cells were pretreated with CsA for $5 \mathrm{~min}$, and then paclitaxel or nocodazole was applied in the presence of CsA. (D) TMRE intensity after 60 min perfusion with an internal solution (CTL, $n=21)$, nocodazole $(1 \mu \mathrm{mol} / \mathrm{L}, \mathrm{n}=8 ; 10 \mu \mathrm{mol} / \mathrm{L}, \mathrm{n}=20)$ and $10 \mu \mathrm{mol} / \mathrm{L}$ nocodazole plus $\mathrm{CsA}(\mathrm{n}=12)$. The protocol was the same as that described in (C). Data are presented as the percentages of TMRE intensity before paclitaxel or nocodazole application, and values are mean \pm SEM. ${ }^{*} \mathrm{P}<0.05$ vs. $C T L,{ }^{\top} \mathrm{P}<0.05$ vs. paclitaxel, $\mathrm{S} P<0.05$ vs. nocodazole by two-way ANOVA and the Bonferroni test. DNP, 2,4-dinitrophenol.

Mitochondria undergo frequent and dynamic morphological changes, and this is regulated by fission and fusion proteins located in the IMM and OMM. ${ }^{12}$ According to recent reports, among these, mitofusin-2 (Mfn2), a fusion protein existing in the OMM, is related to the regulation of mPTP. ${ }^{13}$ Because both Mfn2 and the Miro-Milton-KHC complex are located in the OMM, interaction could occur between these proteins.

In this study, we investigate the relationship between MT disorganization and $\mathrm{mPTP}$ in cardiac myocytes. We used pa- clitaxel and nocodazole, which correspondingly stabilize and disrupt MTs. ${ }^{14}$ Paclitaxel is an important anticancer agent for various solid tumors. However, the cardiotoxic side-effects of paclitaxel, which include rhythmic and conduction disturbances and heart failure, limit the effectiveness of this agent in its clinical use. ${ }^{15}$

We showed that both agents opened MPTP and depolarized mitochondrial membrane potential $(\Delta \Psi \mathrm{m})$, indicating a functional link between MT reorganization and MPTP in cardiac 



Figure 2. Paclitaxel and nocodazole did not alter the mitochondrial membrane potential $(\Delta \psi \mathrm{m})$ of isolated mitochondria. Isolated mitochondria were incubated with or without paclitaxel $(0.1-10 \mu \mathrm{mol} / \mathrm{L})$ or nocodazole $(0.1-10 \mu \mathrm{mol} / \mathrm{L})$ for $60 \mathrm{~min}$. $\Delta \psi \mathrm{m}$ was assessed by JC-1. As a reference, an uncoupler, DNP (100 $\mathrm{mol} / \mathrm{L})$, was applied to promote complete mitochondrial depolarization. (A) The JC-1 ratio after 60 min perfusion with an internal solution $(C T L, n=11)$, paclitaxel $(0.1-10 \mu \mathrm{mol} / \mathrm{L}, \mathrm{n}=11)$ and $\mathrm{DNP}(\mathrm{n}=11)$. (B) The JC-1 ratio after 60 min perfusion with an internal solution (CTL, $n=11)$, nocodazole $(0.1-10 \mu \mathrm{mol} / \mathrm{L}, \mathrm{n}=6)$ and DNP $(n=11)$. Data are presented as the 590-520-nm ratio of JC-1, and values are mean \pm SEM. ${ }^{*}<<0.05$ vs. CTL by two-way ANOVA and the Bonferroni test. JC-1, 5,5',6,6'-tetrachloro-1,1',3,3'-tetraethylbenzimidazolylcarbocyanine iodide; DNP, 2,4-dinitrophenol.

myocytes. We also showed that $\mathrm{Ca}^{2+}$ released from the sarcoplasmic reticulum (SR) plays an important role for the MT-mitochondrial link. These results imply that mitochondria are related to an underlying mechanism by which cardiac function is impaired by MT-disorganization.

\section{Methods}

For further details, refer to Supplementary File 1.

\section{Cell Isolation and Sarcolemmal Membrane Permeabilization}

This investigation conforms to the Guide for the Care and Use of Laboratory Animals published by the US National Institutes of Health (NIH Publication No. 85-23, revised 1996), and the Hamamatsu University School of Medicine Animal Care and Use Committee.

Adult male Sprague-Dawley rats weighing 250-350 g were heparinized (4IU/g i.p.), anaesthetized by an intraperitoneal administration of pentobarbital $(32.4 \mathrm{mg} / \mathrm{kg}$ body weight) and sacrificed by stunning and cervical dislocation. The isolated myocytes were obtained as described previously. ${ }^{16,17}$ After the sarcolemmal membrane permeabilization by saponin $(0.05 \mathrm{mg} / \mathrm{ml})$, the concentration of free calcium $\left(\left[\mathrm{Ca}^{2+}\right]_{\mathrm{c}}\right)$ in an internal solution was changed to $177 \mathrm{nmol} / \mathrm{L}$. $\left[\mathrm{Ca}^{2+}\right]_{\mathrm{c}}$ was obtained by mixing ethylene glycol-bis(2-aminoethylether)-N,N, $\mathrm{N}^{\prime}, \mathrm{N}^{\prime}$-tetraacetic acid (EGTA) and $\mathrm{CaCl}_{2}$, calculated by using a computer program (WINMAXC, provided by Stanford University). All experiments were conducted at room temperature $\left(23^{\circ} \mathrm{C}\right)$ within $6 \mathrm{~h}$ of cell isolation.

\section{Measurements of $\Delta \psi_{\mathrm{m}}$ and mPTP Opening in Permeabilized Myocytes}

The $\Delta \Psi \mathrm{m}$ of permeabilized myocytes was measured with a volt- age fluorescent indicator, tetramethylrhodamine ethyl ester (TMRE, $10 \mathrm{nmol} / \mathrm{L}$ ), and the $\mathrm{mPTP}$ opening was measured with a calcein acetoxymethyl ester (calcein AM, $1 \mu \mathrm{mol} / \mathrm{L}$ ) as described previously. ${ }^{17}$ All measurements were performed with a laser scanning confocal microscope coupled to an inverted microscope (Axiovert 200M, Karl-Zeiss) with a 40× waterimmersion objective lens.

\section{Measurement of $\Delta \psi \mathrm{m}$ in Isolated Mitochondria}

See Supplementary File 1 for details about preparation of isolated mitochondria. The mitochondrial suspension was adjusted to a final concentration of $0.5 \mathrm{mg}$ protein $/ \mathrm{ml}$ with an internal solution, and loaded with $10 \mu \mathrm{mol} / \mathrm{L} \mathrm{JC}-1$ for $30 \mathrm{~min}$ and centrifuged for $20 \mathrm{~min}$ at $7,000 \times \mathrm{g}$. The supernatant was removed and the pellet was re-suspended in an indicator-free internal solution. Then, the mitochondrial suspension was placed in a 96-well plate at $100 \mu \mathrm{l}$ per well. The JC-1 signal was excited at $488 \mathrm{~nm}$ and emission fluorescence was recorded at $528 \mathrm{~nm}$ and $590 \mathrm{~nm}$ with a Synergy HT multi-detection microplatereader (BioTek, Vermont, USA).

\section{Western Blotting}

After drug treatment, permeabilized myocytes were homogenized with a ProteoExtract ${ }^{\circledR}$ Cytosol/Mitochondria Fractionation Kit (Merck Bioscience, Bad Soden, Germany), and then the homogenate was centrifuged for $10 \mathrm{~min}$ at $700 \times g$. The supernatants were subjected to Western blot analysis. Immunoblotting was performed with an enhanced chemiluminescense system (Thermo Fisher Scientific Inc, Massachusetts, USA) according to the manufacturer's instructions. Densitometric analysis was performed using a FUJIFILM LAS 3000-mini (FUJIFILM Corporation, Tokyo, Japan). 


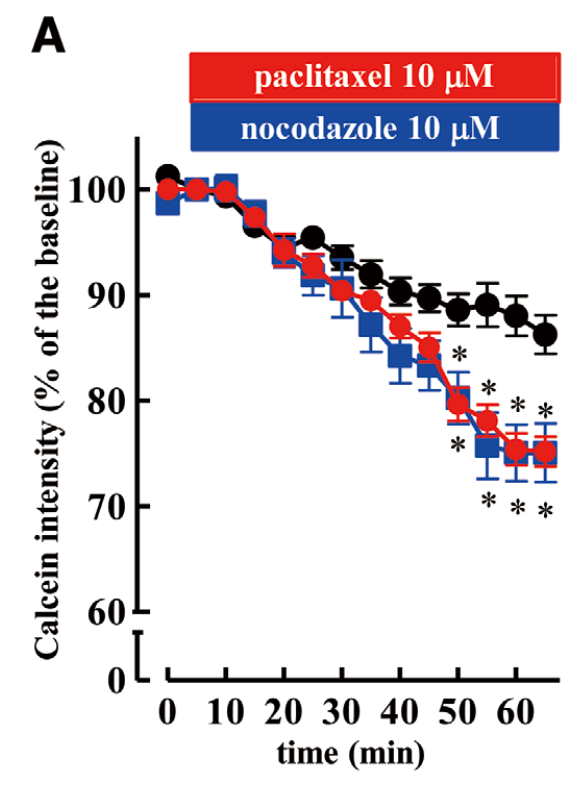

B
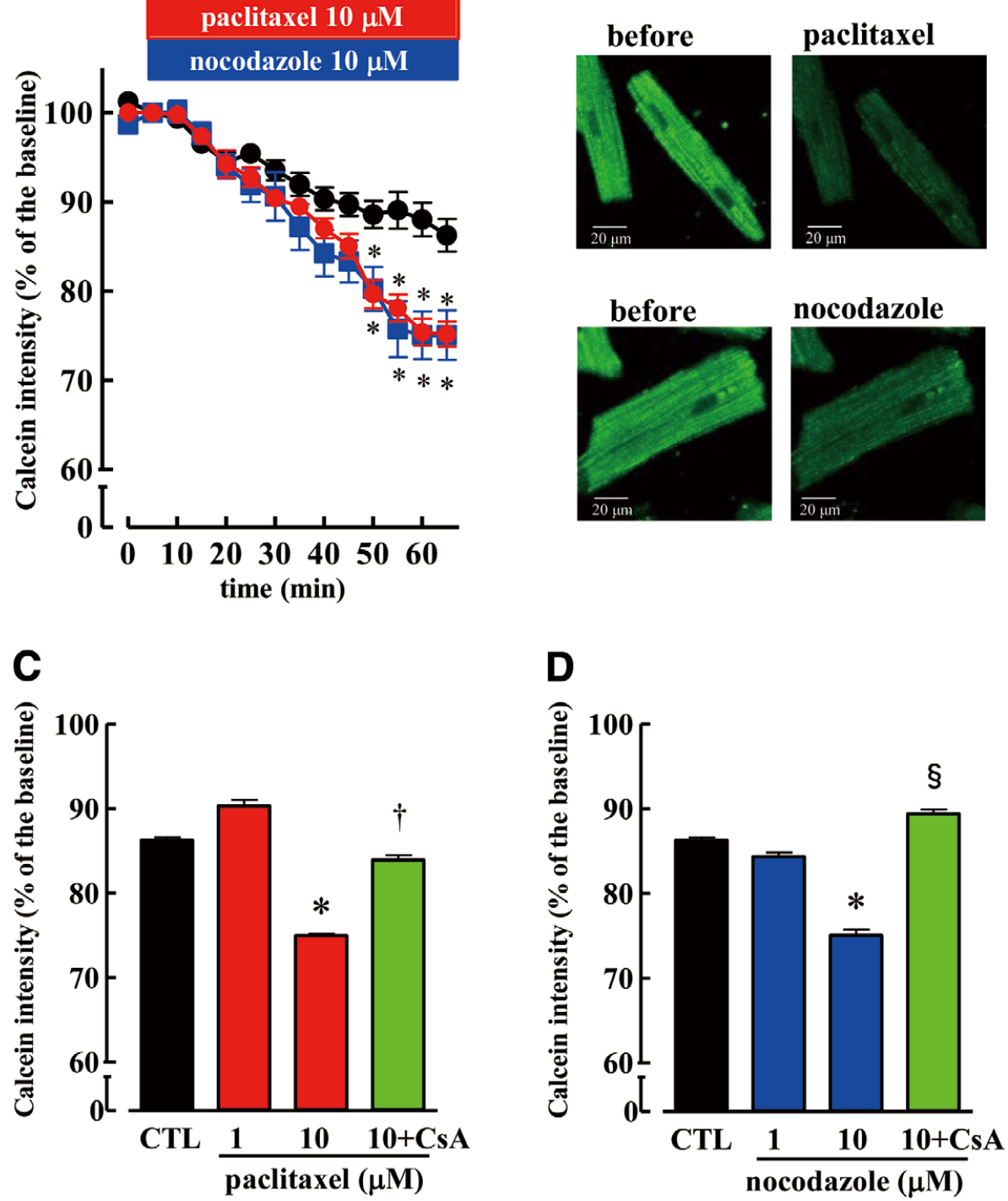

Figure 3. Paclitaxel- and nocodazole-accelerated calcein leakage from mitochondria. (A) Time-courses of the changes in calcein intensity. Permeabilized myocytes were perfused with an internal solution $(\bullet, C T L ; n=27)$ and then paclitaxel $(0,10 \mu \mathrm{mol} / \mathrm{L}$; $\mathrm{n}=48$ ) or nocodazole $(\square, 10 \mu \mathrm{mol} / \mathrm{L}, \mathrm{n}=16)$ was applied. (B) The 2-D images of calcein - Upper left: before paclitaxel; Upper right: after 60 min perfusion of paclitaxel; Lower left: before nocodazole; and Lower right: after 60 min perfusion of nocodazole. (C) Calcein intensity after 60 min perfusion with an internal solution (CTL, $n=27)$, paclitaxel $(1 \mu \mathrm{mol} / \mathrm{L}, \mathrm{n}=9 ; 10 \mu \mathrm{mol} / \mathrm{L}, \mathrm{n}=48), 10 \mu \mathrm{mol} / \mathrm{L}$ paclitaxel plus cyclosporine $A(C s A ; 0.4 \mu \mathrm{mol} / \mathrm{L}, \mathrm{n}=14)$. (D) Calcein intensity after 60 min perfusion with an internal solution (CTL, $n=27)$, nocodazole $(1 \mu \mathrm{mol} / \mathrm{L}, \mathrm{n}=9 ; 10 \mu \mathrm{mol} / \mathrm{L}, \mathrm{n}=16)$, and $10 \mu \mathrm{mol} / \mathrm{L}$ nocodazole plus $\mathrm{Cs} A(n=15)$. In $(\mathbf{C})$ and $(\mathbf{D})$, the protocol for CsA treatment was the same as that described in Figure 1C. Data are presented as the percentages of calcein intensity before paclitaxel or nocodazole application, and values are mean \pm SEM. ${ }^{*} \mathrm{P}<0.05$ vs. $C T L,{ }^{\dagger} \mathrm{P}<0.05$ vs. paclitaxel, $\$ P<0.05$ vs. nocodazole by two-way ANOVA and the Bonferroni test.

\section{Cell Culture and Transfections of H9c2 Cells}

In order to evaluate the mitochondrial morphology, the H9c2 cells were transfected with mitochondrial yellow fluorescent proteins (YFP) (Clontec Laboratories, Inc, California, USA). Transfection of the H9c2 cells was performed using Lipofectamine ${ }^{\circledR}$ LTX, according to the manufacturer's protocol (Life Technologies, California, USA). The experiments were conducted $72 \mathrm{~h}$ after transfection according to the study protocol.

\section{Chemicals}

Nocodazole, cyclosporin A (CsA), 1,2-Bis(2-aminophenoxy) ethane-N,N,N',N'-tetraacetic acid tetrasodium salt (BAPTA), thapsigargin (TG) and 2-aminoethoxydiphenyl borate (2APB) were purchased from Sigma-Aldrich (Michigan, USA). Ru360 was purchased from Calbiochem-Novabiochem Corp. (California, USA). Paclitaxel was purchased from Enzo Life Sciences (Pennsylvania, USA). All fluorescent dyes were purchased from Life Technologies (California, USA). 


\section{Data Analyses}

Data are presented as means \pm SEM, and the number of cells or experiments is shown as $n$. Statistical analyses were performed using the $t$-test or two-way ANOVA of repeated measurements, followed by the Bonferroni test. A level of $\mathrm{P}<0.05$ was accepted as statistically significant.

\section{Results}

\section{MT Disorganization Depolarizes $\Delta \psi \mathrm{m}$}

In the preliminary experiments, dose-dependent effects of paclitaxel and nocodazole on MTs were examined in permeabilized myocytes. As shown in Figures S1C and S1E, paclitaxel $(10 \mu \mathrm{mol} / \mathrm{L})$ or nocodazole $(10 \mu \mathrm{mol} / \mathrm{L})$ correspondingly stabilizes and disrupts MTs. One $\mu \mathrm{mol} / \mathrm{L}$ of paclitaxel or nocodazole did not alter MT organization (Figures S1B,D). From these data, we used $10 \mu \mathrm{mol} / \mathrm{L}$ paclitaxel or $10 \mu \mathrm{mol} / \mathrm{L}$ nocodazole in the following experiments. Next, we investigated the effects of paclitaxel and nocodazole on mitochondrial morphology by using electron microscope (EM). Paclitaxel increased mitochondrial swelling and vacuolar formation. In contrast, nocodazole disturbed $\mathrm{Z}$ line configuration and caused disarrangement of mitochondria (Figure S2).

Figure 1A shows the time-courses of changes in TMRE intensity, which represents the changes in $\Delta \Psi \mathrm{m}$, during and after the application of paclitaxel or nocodazole. Paclitaxel or nocodazole significantly depolarized $\Delta \Psi \mathrm{m}$ (CTL, $88.3 \pm 3.6 \%$ of the baseline; paclitaxel, $57.5 \pm 5.1 \%$ of the baseline, $\mathrm{P}<0.05$ vs. CTL. Nocodazole, $63.1 \pm 4.7 \%$ of the baseline, $\mathrm{P}<0.05$ vs. CTL). To confirm whether $\Delta \Psi \mathrm{m}$ depolarization by each drug was related to MPTP, the effect of a mPTP inhibitor, cyclosporin A (CsA, $0.4 \mu \mathrm{mol} / \mathrm{L})$, on $\Delta \Psi \mathrm{m}$ depolarization was investigated. When cells were pretreated with CsA, the reduction of TMRE intensity induced by each drug was cancelled (CsA, 92.8 $\pm 4.5 \%$ of the baseline, $\mathrm{P}<0.05$ vs. paclitaxel alone, $88.7 \pm 5.2 \%$ of the baseline, $\mathrm{P}<0.05$ vs. nocodazole alone).

\section{Effects of Paclitaxel and Nocodazole on $\Delta \psi \mathrm{m}$ of Isolated Mitochondria}

To examine the direct effects of paclitaxel and nocodazole on mitochondria, a suspension of isolated mitochondria was incubated with or without paclitaxel $(0.1-10 \mu \mathrm{mol} / \mathrm{L})$ or nocodazole $(0.1-10 \mu \mathrm{mol} / \mathrm{L})$ for $60 \mathrm{~min}$, and the $\Delta \Psi \mathrm{m}$ was assessed by JC- 1 fluorescence. As shown in Figures $2 \mathrm{~A}$ and $2 \mathrm{~B}$, neither paclitaxel nor nocodazole depolarized the $\Delta \Psi \mathrm{m}$ of isolated mitochondria. These results indicate that paclitaxel- or nocodazoleinduced depolarization of $\Delta \Psi \mathrm{m}$ is not due to the direct effect of each agent on the mitochondria.

\section{Effects of Paclitaxel and Nocodazole on mPTP}

The effects of paclitaxel and nocodazole on MPTP were examined using fluorescent calcein. The loading of calcein-AM before the permeabilization of myocytes facilitated the localization of calcein into mitochondria. This mitochondria-entrapped calcein was released when mPTP opened. Thus, the reduction of the calcein signal indicates mPTP opening. ${ }^{17}$ As shown in Figure 3A, application of paclitaxel or nocodazole decreased calcein intensity significantly (CTL, $86.3 \pm 1.8 \%$ of the baseline; paclitaxel, $75.0 \pm 1.4 \%$ of the baseline, $\mathrm{P}<0.05$ vs. CTL; nocodazole, $75.1 \pm 2.8 \%$ of the baseline, $\mathrm{P}<0.05$ vs. CTL). The opening of $\mathrm{mPTP}$ by each drug was inhibited by CsA $(0.4 \mu \mathrm{mol} / \mathrm{L}, 83.9 \pm 2.1 \%$ of the baseline, $\mathrm{P}<0.05 \mathrm{vs}$. paclitaxel alone; $89.4 \pm 2.1 \%$ of the baseline, $\mathrm{P}<0.05$ vs. nocodazole alone). During the perfusion of paclitaxel or nocodazole, there were no changes in cell shape, possibly due to the continuous perfu- sion of ATP, and loss of apoptosis-related signaling molecules in our experimental condition.

\section{Intracellular $\mathrm{Ca}^{2+}$ and Paclitaxel- or Nocodazole-Induced mPTP Opening}

Because intracellular $\mathrm{Ca}^{2+}$ is involved in the regulation of $\mathrm{mPTP}$, we investigated the role of intracellular $\mathrm{Ca}^{2+}$ for paclitaxel- or nocodazole-induced mPTP opening. As shown in Figures 4A and $4 \mathrm{C}$, when BAPTA $(5 \mathrm{mmol} / \mathrm{L})$ was added to the solution for the complete chelation of $\mathrm{Ca}^{2+}$, paclitaxel-induced mPTP opening was inhibited (paclitaxel with BAPTA, $86.0 \pm 1.3 \%$ of the baseline, $\mathrm{P}<0.05$ vs. paclitaxel with $177 \mathrm{nmol} / \mathrm{L} \mathrm{Ca}^{2+}, 75.0 \pm 1.4 \%$ of the baseline). In contrast, nocodazole-induced mPTP opening was not inhibited (Figures 4B,D; nocodazole with BAPTA, $69.3 \pm 2.8 \%$ of the baseline; nocodazole with $177 \mathrm{nmol} / \mathrm{L}$ $\mathrm{Ca}^{2+}, 75.1 \pm 2.8 \%$ of the baseline). In the nominal $\mathrm{Ca}^{2+}$-free $\left(\left[\mathrm{Ca}^{2+}\right]_{\mathrm{c}}=0 \mathrm{nmol} / \mathrm{L}+\mathrm{EGTA} 3 \mathrm{mmol} / \mathrm{L}\right.$; labeled $\left[\mathrm{Ca}^{2+}\right]_{\mathrm{c}}=0$ in the graph) internal solution, paclitaxel and nocodazole opened mPTP as much as those observed in the control solution $\left(\left[\mathrm{Ca}^{2+}\right]_{\mathrm{c}}=177 \mathrm{nmol} / \mathrm{L}\right)\left(\right.$ paclitaxel with $0 \mathrm{nmol} / \mathrm{L} \mathrm{Ca}^{2+}, 69.6 \pm 2.2 \%$ of the baseline; nocodazole with $0 \mathrm{nmol} / \mathrm{L} \mathrm{Ca}^{2+}, 65.9 \pm 2.3 \%$ of the baseline; Figures 4C,D). These results suggest that paclitaxel-induced mPTP opening requires a local intracellular $\mathrm{Ca}^{2+} .18$

\section{Sarcoplasmic Reticulum Ca ${ }^{2+}$ Release and Paclitaxel- or Nocodazole-Induced mPTP Opening}

MTs are closely associated with mitochondria and the SR. ${ }^{6} \mathrm{We}$ investigated the effects of TG, which inhibits sarcoplasmic reticulum $\mathrm{Ca}^{2+}$-ATPase (SERCA) and empties the $\mathrm{SR} \mathrm{Ca}^{2+}$ content, on paclitaxel- or nocodazole-induced mPTP opening. When cells were pretreated with TG $(10 \mu \mathrm{mol} / \mathrm{L})$, paclitaxel-induced $\mathrm{mPTP}$ opening was inhibited $(84.2 \pm 2.1 \%$ of the baseline, $\mathrm{n}=21$, $\mathrm{P}<0.05$ vs. without TG; Figure 5A). However, the nocodazoleinduced $\mathrm{mPTP}$ opening was not inhibited by TG $(75.3 \pm 3.8 \%$ of the baseline; Figure 5B).

Inositol 1,4,5-trisphosphate receptor (InsP3R) is one of the $\mathrm{Ca}^{2+}$ release channels expressed in cardiac myocytes. ${ }^{19} \mathrm{In}$ neonatal cardiomyocytes, paclitaxel accelerates spontaneous $\mathrm{Ca}^{2+}$ oscillations via InsP3R. ${ }^{20}$ Thus, we investigated whether paclitaxel-induced mPTP opening was dependent on InsP3R. For this purpose, 2-aminoethoxydiphenyl borate (2-APB; $20 \mu \mathrm{mol} / \mathrm{L}$ ), a blocker of InsP3R, was used. As shown in Figure 5C, pretreatment of 2-APB did not inhibit the effects of paclitaxel on mPTP opening (74.2 $\pm 1.5 \%$ of the baseline). These results suggest that $\mathrm{Ca}^{2+}$ released from the SR is required for paclitaxel-induced, but not for nocodazole-induced mPTP opening. It is also indicated that $\mathrm{Ca}^{2+}$ release by InsP3R is not involved in paclitaxel-induced mPTP opening in adult cardiac myocytes.

\section{Mitochondrial $\mathrm{Ca}^{2+}$ Uptake is not Required for MT Disorganization-Induced mPTP Opening}

Mitochondrial $\mathrm{Ca}^{2+}$ is one of the key inducers of mPTP. Because the SR Ca ${ }^{2+}$-releasing site and mitochondria are located within a highly restricted microdomain, $\mathrm{Ca}^{2+}$ released from the $\mathrm{SR}$ could be taken up into the mitochondria and cause MPTP to open. We examined the effect of Ru360, an inhibitor of the mitochondrial $\mathrm{Ca}^{2+}$ uniporter, on paclitaxel- or nocodazoleinduced mPTP opening. As shown in Figures 5D and 5E, the effects of paclitaxel $(72.8 \pm 1.2 \%$ of the baseline) or nocodazole $(68.9 \pm 3.7 \%$ of the baseline) on mPTP opening were not inhibited by Ru360. 

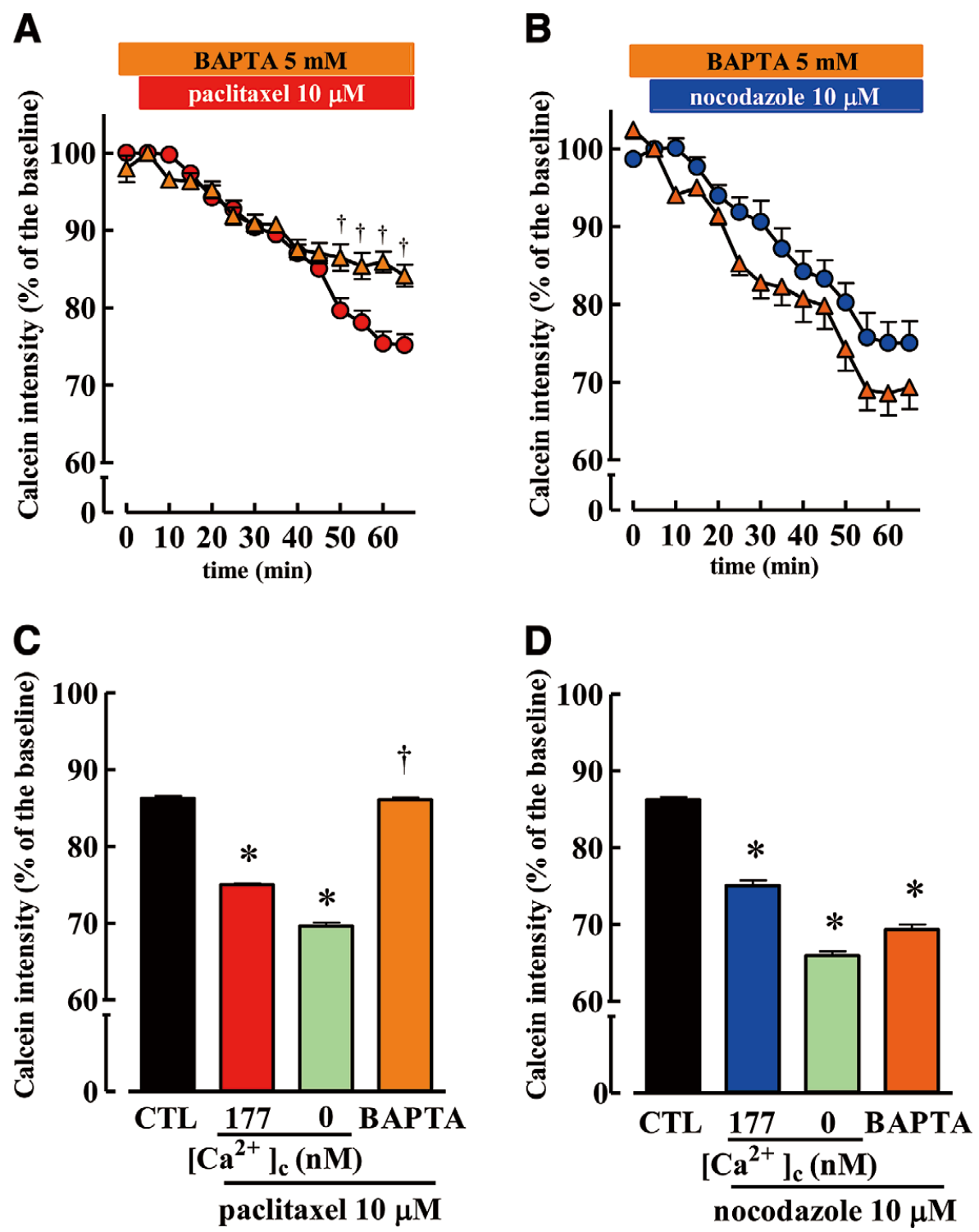

Figure 4. Intracellular $\mathrm{Ca}^{2+}$ affected the effect of paclitaxel, but not the effect of nocodazole, on mPTP opening. (A) The timecourses of the changes in calcein intensity after $60 \mathrm{~min}$ perfusion with paclitaxel $(10 \mu \mathrm{mol} / \mathrm{L})$ in an internal solution with $177 \mathrm{nmol} / \mathrm{L}$ $\mathrm{Ca}^{2+}(\mathrm{O}, \mathrm{n}=48)$ or $\left[\mathrm{Ca}^{2+}\right]_{\mathrm{c}}=0 \mathrm{nmol} / \mathrm{L}+\mathrm{BAPTA} 5 \mathrm{mmol} / \mathrm{L}(\triangle, \mathrm{n}=21)$. (B) The same experimental protocol as $(\mathbf{A})$ was performed with nocodazole $(10 \mu \mathrm{mol} / \mathrm{L})$ in an internal solution with $177 \mathrm{nmol} / \mathrm{L} \mathrm{Ca}{ }^{2+}(\mathbf{O}, \mathrm{n}=16)$ or $\left[\mathrm{Ca}^{2+}\right]_{\mathrm{c}}=0 \mathrm{nmol} / \mathrm{L}+\mathrm{BAPTA}(\triangle, \mathrm{n}=18)$. (C and $\left.\mathbf{D}\right)$ Calcein intensity after 60 min perfusion with paclitaxel (C) and nocodazole (D). Data are presented as the percentages of calcein intensity before paclitaxel or nocodazole application, and values are mean \pm SEM. CTL, calcein intensity after 60 min perfusion with an internal solution with $177 \mathrm{nmol} / \mathrm{L} \mathrm{Ca}{ }^{2+}(\mathrm{n}=27)$. $\left[\mathrm{Ca}^{2+}\right]_{\mathrm{c}}=0: \mathrm{Ca}^{2+}$-free $\left(\left[\mathrm{Ca}^{2+}\right]_{\mathrm{c}}=0 \mathrm{nmol} / \mathrm{L}+\mathrm{EGTA} 3 \mathrm{mmol} / \mathrm{L}\right)$. Paclitaxel with $0 \mathrm{nmol} / \mathrm{L}$ $\mathrm{Ca}^{2+} ; \mathrm{n}=23$, nocodazole with $0 \mathrm{nmol} / \mathrm{L} \mathrm{Ca}^{2+} ; \mathrm{n}=16 .{ }^{*} \mathrm{P}<0.05$ vs. CTL, ${ }^{\mathrm{P}} \mathrm{P}<0.05$ vs. paclitaxel by two-way ANOVA and the Bonferroni test. BAPTA, 1,2-Bis(2-aminophenoxy)ethane- $N, N, N^{\prime}, N^{\prime}$-tetraacetic acid tetrasodium salt; EGTA, ethylene glycol-bis(2-

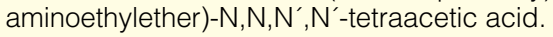

\section{Paclitaxel-Induced mPTP Opening is Related to the Reduction of Mfn2}

The mitochondrial Rho-GTPase (Miro), which possesses a $\mathrm{Ca}^{2+}$ binding EF-hands domain, is involved in the OMM complex, which connects mitochondria to MTs. Miro is known to interact with a mitochondrial fusion protein, Mfn2. ${ }^{21}$ Because there is a functional link between Mfn2 and $\mathrm{mPTP},{ }^{13}$ we investigated the effect of paclitaxel or nocodazole on Mfn2 expression. As shown in Figures $\mathbf{6 A}$ and $6 \mathrm{~B}$, the treatment of permeabilized myocytes with paclitaxel $(10 \mu \mathrm{mol} / \mathrm{L})$ for $60 \mathrm{~min}$ reduced the Mfn2 expression $(0.67 \pm 0.064 \mathrm{AU}$ [arbitrary units], $\mathrm{n}=9, \mathrm{P}<0.05$ vs. control), and this reduction of Mfn2 by paclitaxel was inhibited in the presence of BAPTA $(0.83 \pm 0.075 \mathrm{AU}, \mathrm{n}=9$, n.s. vs. control with BAPTA). EGTA did not alter the Mfn2 expression after paclitaxel treatment.

These data suggest that the SR-released $\mathrm{Ca}^{2+}$ is also required for the effect of paclitaxel on the expression of mitochondrial fusion protein. 

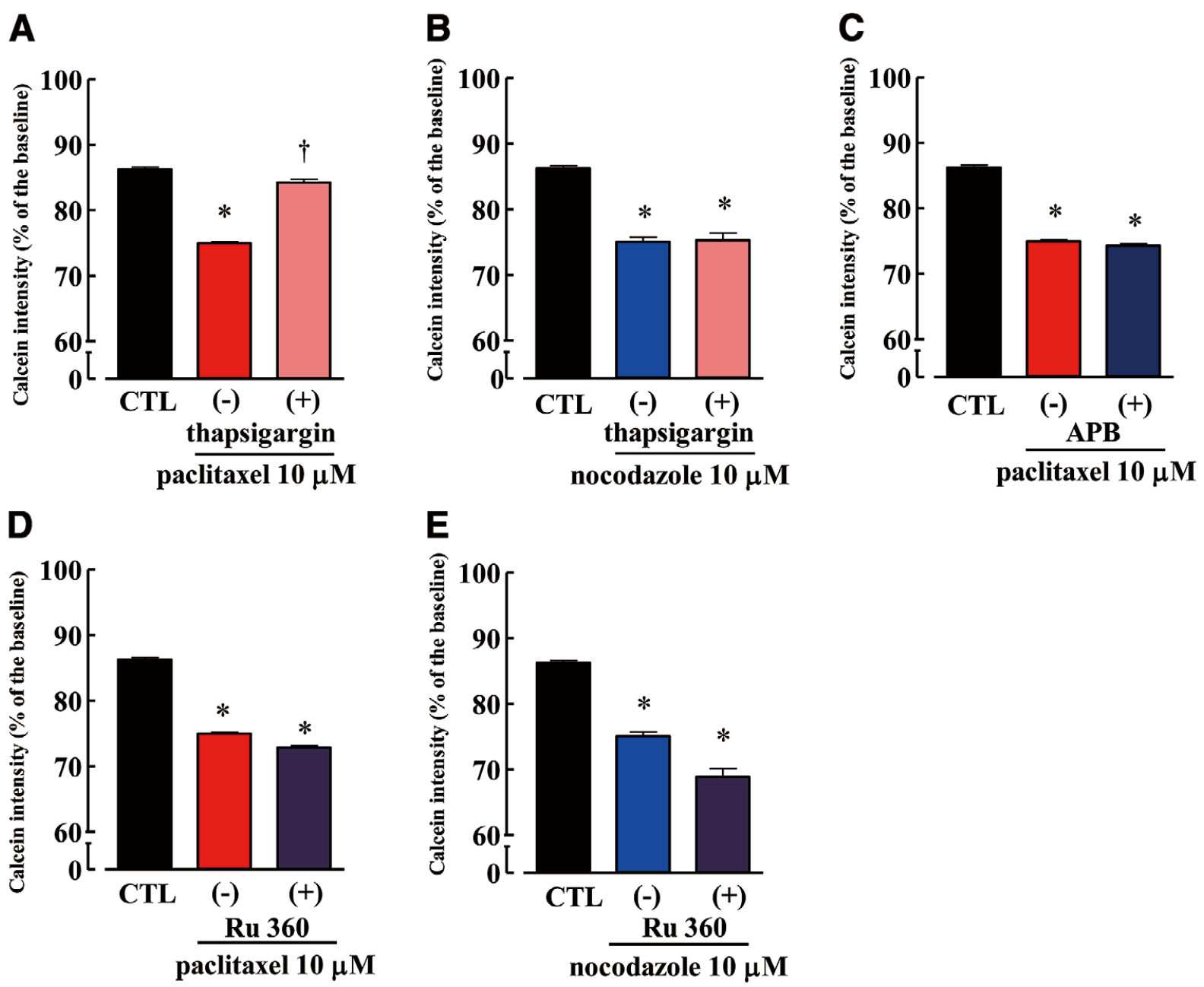

Figure 5. The sarcoplasmic reticulum (SR) $\mathrm{Ca}^{2+}$ was involved in the effects of paclitaxel but not in the effects of nocodazole on mPTP. (A and B) Cells were exposed to thapsigargin (TG; $10 \mu \mathrm{mol} / \mathrm{L}$ ) for $15 \mathrm{~min}$ before the application of paclitaxel or nocodazole to diminish SR Ca ${ }^{2+}$. (A) Calcein intensity after 60 min perfusion with an internal solution (CTL, $\left.n=27\right)$, or paclitaxel $(10 \mu \mathrm{mol} / \mathrm{L})$ with $(n=21)$ or without TG $(n=48)$. (B) The same protocol as $(A)$ was conducted with nocodazole $(10 \mu \mathrm{mol} / \mathrm{L})$ with $(n=11)$ or without TG $(n=16)$. (C) Calcein intensity after 60 min perfusion with an internal solution (CTL, $n=27)$, paclitaxel $(10 \mu$ mol/L) with $(n=25)$ or without $(n=48)$ Ins3PR blocker; 2-APB (10 $\mu \mathrm{mol} / \mathrm{L})$. 2-APB was applied for $5 \mathrm{~min}$ before the perfusion of paclitaxel. (D and $\mathbf{E})$ Cells were pretreated with an inhibitor of mitochondrial $\mathrm{Ca}^{2+}$ uniporter, Ru360 $(10 \mu \mathrm{mol} / \mathrm{L})$ for $5 \mathrm{~min}$ and then paclitaxel or nocodazole was applied in the presence of Ru360. (D) The summarized data of calcein intensity after 60 min perfusion with an internal solution (CTL, $n=27)$, paclitaxel $(10 \mu \mathrm{mol} / \mathrm{L})$ with $(n=19)$ or without Ru360 $(n=48)$. (E) Calcein intensity after 60 min perfusion with an internal solution (CTL, $n=27)$, nocodazole $(10 \mu \mathrm{mol} / \mathrm{L})$ with $(n=9)$ or without Ru360 $(n=16)$. Data are presented as the percentages of calcein intensity before the application of paclitaxel or nocodazole, and values are mean $\pm S E M$. ${ }^{*} \mathrm{P}<0.05 \mathrm{vs}$. $\mathrm{CTL}$, ${ }^{\mathrm{P}} \mathrm{P}<0.05 \mathrm{vs}$. paclitaxel by two-way ANOVA and the Bonferroni test. Ins3PR, inositol 1,4,5-triphosphate receptor; 2-APB, 2-aminoethoxydiphenyl borate.

\section{MT Disorganization Induces Mitochondrial Fragmentation in H9c2 Cells}

Recent studies have reported that Mfn2 deficiency resulted in mitochondrial fragmentation in cultured myocytes..$^{13,22}$ Thus, we examined the effects of MT disorganization caused by paclitaxel on mitochondrial morphology in $\mathrm{H} 9 \mathrm{c} 2$ cells. Figure $6 \mathrm{C}$ shows the immunofluorescent staining of mito-YFP transfected $\mathrm{H} 9 \mathrm{c} 2$ cells with monoclonal anti $\alpha$-tubulin antibodies. In H9c2 cells, the immunofluorescent staining of anti $\alpha$-tubulin antibodies showed that paclitaxel $(10 \mu \mathrm{mol} / \mathrm{L})$ increased MT network intensity, as in the case of adult myocytes. As shown in Figure 6D, paclitaxel induced mitochondrial fragmentation significantly (67.6 $\pm 2.2 \%$ of cells, $\mathrm{P}<0.05$ vs. control) compared with the control (14.5 $\pm 1.6 \%$ of cells), and this effect was not inhibited by $\mathrm{CsA}$.

\section{Discussion}

\section{Disruption of the MT Network-Opened mPTP}

We demonstrated that nocodazole, which disrupts MTs and thus destroys the connection between MTs and mitochondria ${ }^{7}$, depolarized $\Delta \Psi \mathrm{m}$ and opened $\mathrm{mPTP}$. MT disruption-induced mPTP opening was not affected by cytosolic $\mathrm{Ca}^{2+}$. In line with our results, disruption of the MT network disorganized the regular arrangement of mitochondria, and this was accompanied by a decrease in mitochondrial respiration. ${ }^{23}$ These results in- 

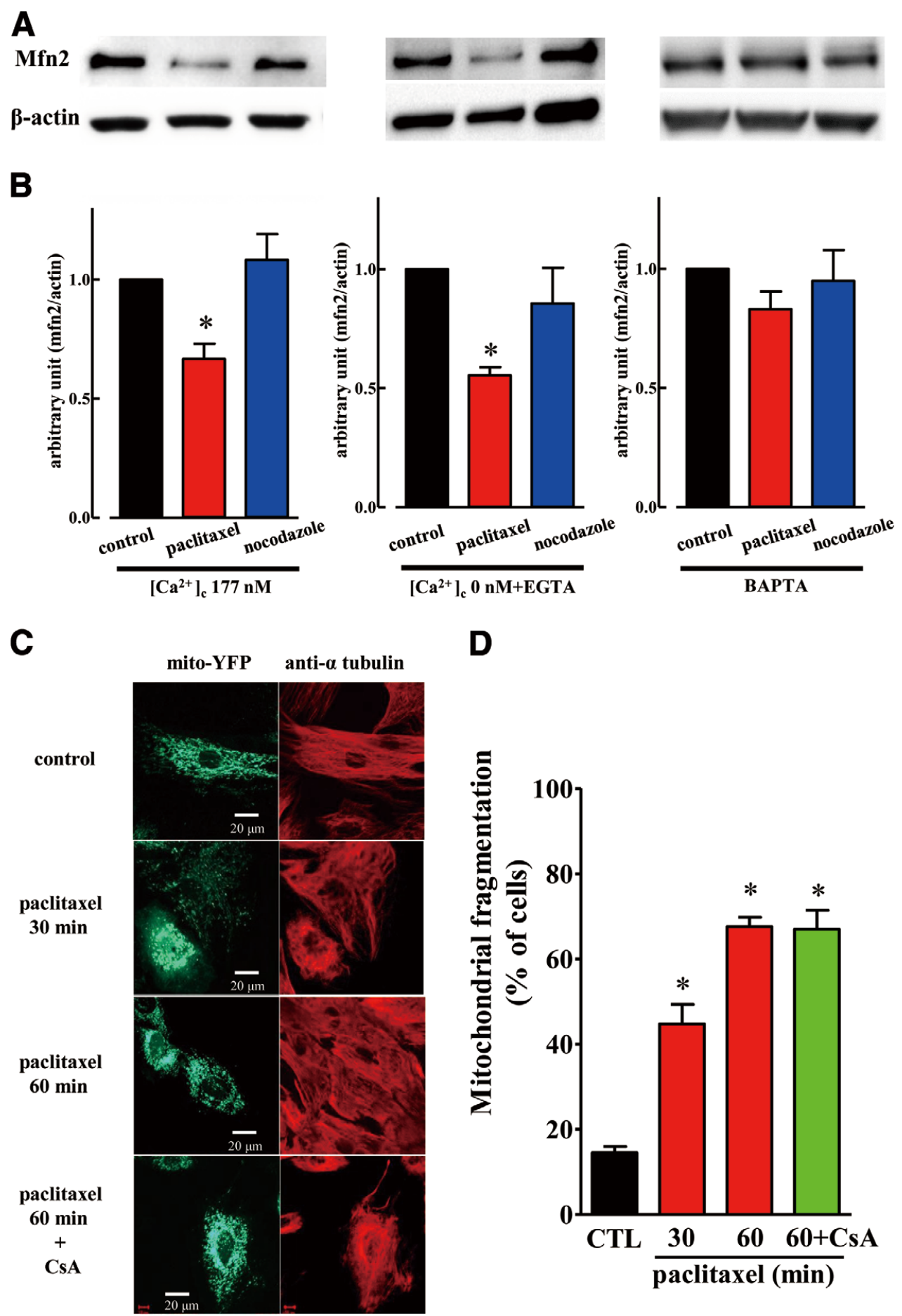

D

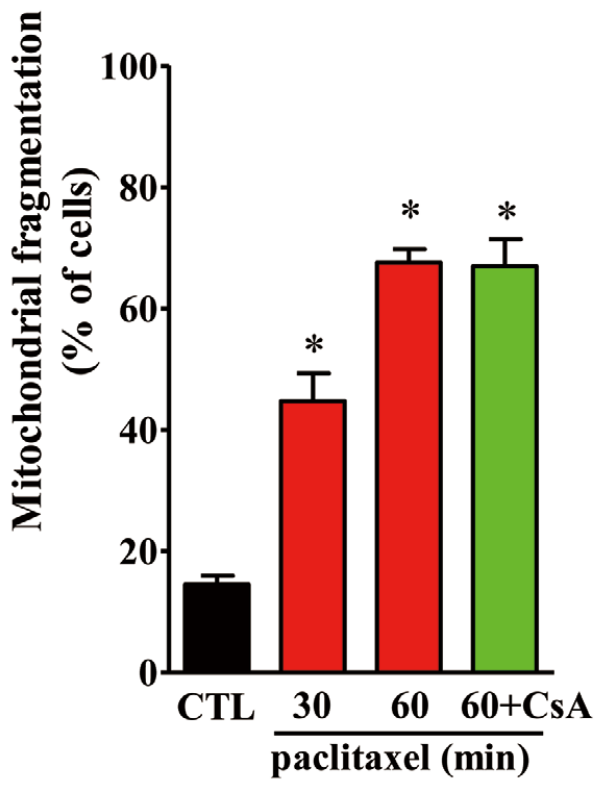

Figure 6. Paclitaxel decreased protein levels of mitofusion-2 (Mfn2) in myocytes and induced mitochondrial fragmentation. (A and B) Cells were incubated with or without paclitaxel $(10 \mu \mathrm{mol} / \mathrm{L})$ or nocodazole $(10 \mu \mathrm{mol} / \mathrm{L})$ for $60 \mathrm{~min}$ in an internal solution with $177 \mathrm{nmol} / \mathrm{L}$ $\mathrm{Ca}^{2+}$ or nominally zero $\mathrm{Ca}^{2+}+$ EGTA (3mmol/L) or BAPTA (5mmol/L). (A) Representative Western blots of the expression of Mfn2 proteins after the incubation of myocytes for 60 min with each drug. (B) The summarized data of Mfn2 intensity. Western blot data were normalized to $\beta$-actin and expressed as fold-change compared with control with or without EGTA or BAPTA; values are mean \pm SEM; control, $n=9$; paclitaxel, $n=9$; nocodazole, $n=9$; control with EGTA, $n=6$; paclitaxel with $E G T A, n=6$; nocodazole with $E G T A$, $n=6$; control with BAPTA, $n=9$; paclitaxel with BAPTA, $n=9$; nocodazole with BAPTA, $n=9$. ${ }^{*} P<0.05$ vs. control. (C and $\left.\mathbf{D}\right)$ H9c2 cells were incubated with a control solution or paclitaxel $(10 \mu \mathrm{mol} / \mathrm{L})$ with or without CsA $(0.1 \mu \mathrm{mol} / \mathrm{L})$. (C) The immunofluorescent staining of mito-YFP-transfected H9c2 cells with monoclonal anti $a$-tubulin antibodies. (D) Summarized data of percentage of cells that showed mitochondrial fragmentation in each condition. Data are presented as the percentage of total cells. ${ }^{*} \mathrm{P}<0.05$ vs. CTL by two-way ANOVA and the Bonferroni test. BAPTA, 1,2-Bis(2-aminophenoxy)ethane-N,N,N',N'-tetraacetic acid tetrasodium salt; EGTA, ethylene glycol-bis(2-aminoethylether)$\mathrm{N}, \mathrm{N}, \mathrm{N}^{\prime}, \mathrm{N}^{\prime}$-tetraacetic acid; YFP, yellow fluorescent proteins. 
dicate that intact MT-mitochondria co-localization is required to maintain mitochondrial function.

It should be considered that disruption of MTs results in the increase of tubulin, which interacts with the voltage-dependent anion channel (VDAC) located in the OMM;24 modification of VDAC has been known to regulate mPTP. ${ }^{5}$ Because we used permeabilized myocytes, cytosolic tubulin would be washed out from the cytosol to the extracellular space under our experimental conditions.

\section{Stabilization of MTs and mPTP}

Although previous studies have reported that paclitaxel opens mPTP, $, 25,26$ the mechanism by which it accomplishes this has not been fully explored. Using isolated neuroblastoma mitochondria, Andre et al. demonstrated direct and cyclosporin A-sensitive effects of paclitaxel on mPTP. ${ }^{26}$ However, no direct effect of paclitaxel on mitochondria was observed in our experiment.

We have shown that paclitaxel-induced mPTP opening was inhibited by the complete chelation of cytosolic $\mathrm{Ca}^{2+}$ or by the depletion of the $\mathrm{SR} \mathrm{Ca}^{2+}$, suggesting that $\mathrm{Ca}^{2+}$ released from the SR is involved in the signal transduction cascade. The SRreleased $\mathrm{Ca}^{2+}$ dominates $\mathrm{Ca}^{2+}$ concentration in the very restricted microdomain between mitochondria and SR, and influences mitochondrial $\mathrm{Ca}^{2+}$ homeostasis. ${ }^{18}$ Thus, it is possible that paclitaxel alters $\mathrm{Ca}^{2+}$ release from the SR and subsequently affects the regulation of $\mathrm{mPTP}$ by altering mitochondrial $\mathrm{Ca}^{2+}$ homeostasis.

In our study, paclitaxel did not alter the frequency, duration and the amplitude of $\mathrm{Ca}^{2+}$ sparks, which represent the SR $\mathrm{Ca}^{2+}$ releases from ryanodine receptors (Figure S3). This is in contrast to the previous result that $\mathrm{Ca}^{2+}$ releases from the $\mathrm{SR}$ were inhibited by paclitaxel. ${ }^{27}$ Differences in the experimental protocol might account for these discrepancies. In neonatal rat ventricular myocytes, paclitaxel accelerated spontaneous oscillatory changes in cytosolic $\mathrm{Ca}^{2+}$ by interacting with InsP3R. ${ }^{20}$ The effect of paclitaxel on mPTP was not blocked by the inhibiter of InsP3R in this study. $\mathrm{Ca}^{2+}$-releasing channels from the SR are different between neonatal and adult ventricular myocytes, and $\mathrm{Ca}^{2+}$ release by InsP3R is not dominant in adult ventricular myocytes.

It should also be considered that paclitaxel or nocodazole could increase reactive oxygen species (ROS) production, and that ROS affects the opening of mPTP. Although paclitaxel and nocodazole increased ROS generation, the effect of ROS on mPTP was observed only when paclitaxel was used (Figure S4). It is known that mitochondrial fragmentation is also related to ROS production. ${ }^{12}$ Thus, synergistic effects on the generation of ROS by mPTP and mitochondrial fragmentation could lead to positive feedback about mPTP opening. In contrast, structural destruction than ROS may have a larger impact on nocodazole-induced mPTP opening. Further study is required to clarify the relationship among MTs, ROS generation and $\mathrm{mPTP}$ opening.

Finally, because both paclitaxel and nocodazole have been known to alter glycogen synthase kinase 3 beta (GSK3 $\beta$ ) activity, which is an upstream signaling mechanism to regulate mPTP, in addition to the direct effect of paclitaxel or nocodazole on MTs, it is possible that paclitaxel or nocodazole regulates $\mathrm{mPTP}$, at least in part, via intracellular signaling cascades, such as GSK $3 \beta .{ }^{28,29}$

\section{Effects of Paclitaxel on the MPTP and OMM Complex}

In this study, the inhibition of mitochondrial $\mathrm{Ca}^{2+}$ uptake by Ru360 did not affect paclitaxel-induced mPTP opening, which is different from previous findings. ${ }^{18}$ This led us to the hypothesis that a mechanism other than mitochondrial $\mathrm{Ca}^{2+}$-uptake could be involved to cause the paclitaxel-induced mPTP opening.

The connection of mitochondria to MTs comprises the OMM complex, also called the Miro-Milton-KHC system. This involves the OMM protein, Miro, which indirectly attaches to KHC through the adaptor protein, Milton. ${ }^{10}$ Miro possesses $\mathrm{Ca}^{2+}$-sensing EF-hand motifs and regulates mitochondrial movement along MTs. ${ }^{11}$ This implies the possibility that SR-released $\mathrm{Ca}^{2+}$ is sensed by Miro, and the activated signal is conveyed to mitochondria.

Mitochondria undergo dynamic morphological changes by fission and fusion. The mitochondrial fusion is regulated by Mfn2 located on the OMM and optic atrophy 1 (Opa1) located on the IMM. ${ }^{12}$ In the neuron system, Mfn2 directly interacts with Miro, and both Mfn2 and Miro are required to cooperatively mediate mitochondrial transport. ${ }^{21}$ Miro can regulate mitochondrial morphology, independently from mitochondrial transport in H9c2 cells. ${ }^{6}$ Here, we showed that paclitaxel-induced $\mathrm{mPTP}$ opening was associated with the reduction of Mfn2 expression, and that paclitaxel increased the fraction of small mitochondria and this mitochondrial fragmentation was not inhibited by CsA. These imply that mitochondrial fission was accelerated by paclitaxel and might precede mPTP opening. In $\mathrm{H} 9 \mathrm{c} 2$ cells, mitochondrial fragmentation was accompanied with the depolarization of $\Delta \Psi \mathrm{m}$ (Figure S5). Thus, both mPTP opening and fragmentation affect $\Delta \Psi \mathrm{m}$ in $\mathrm{H} 9 \mathrm{c} 2$ cells. Interestingly, as shown in Figure $\mathbf{S}$ 2, paclitaxel caused swollen and vacuolar-formed mitochondria in permeabilized ventricular myocytes, which are consistent with previous results obtained from Mfn2 knockout mouse. ${ }^{13,30}$ The reason for the differences of Mfn2-mediated changes in mitochondrial morphology between $\mathrm{H} 9 \mathrm{c} 2$ cells and adult myocytes remains to be resolved. Taken together, it is suggested that the modification of MTmitochondrial connections activates OMM complex-medicated signaling and influences mitochondrial dynamic protein, resulting in the opening of $\mathrm{MPTP}$ and mitochondrial dysfunction. In contrast to our results, Papanicolaou et al. reported that there was a delay of mPTP opening in cardiac myocytes obtained from Mfn2 knockout mouse. ${ }^{13}$ The different animal species or experimental environments, such as ischemia/reperfusion might account for the discrepancies. Further investigations are required to clarify the mechanisms by which interactions between the OMM complex and mitochondrial dynamic proteins are regulated.

Mfn2 is also involved in ER-mitochondrial tethering. ${ }^{31}$ Thus, the reduction of Mfn 2 could influence $\mathrm{Ca}^{2+}$ homeostasis in a very restricted space around the mitochondria by altering SR-mitochondrial co-localization, ${ }^{30}$ and then have some impact on paclitaxel-induced mPTP opening.

\section{Clinical Implication}

Disruption of MTs occurs in the early stage of ischemia and contributes to ischemic myocardial injury. ${ }^{32}$ Changes in MT density affects cardiac contractility or $\mathrm{Ca}^{2+}$ handling, ${ }^{27,33}$ and is implicated in the pathogenesis of hypertrophic heart disease or heart failure., ${ }^{2,3}$ Moreover, adverse cardiac effects of paclitaxel in impairing cardiac function have been observed during the cancer treatment of patients treated with paclitaxel. ${ }^{15}$ In addition to previously proposed mechanical or electrical mechanisms, modulation of mPTP, and thus cardiac metabolism, could account for cardiac dysfunction caused by MT disorganization. 


\section{Conclusions}

Our study provides evidence that MT disorganization plays an important role in the regulation of $\mathrm{mPTP}$ in cardiac myocytes. For MT-mitochondria interaction, intact MT-mitochondria co-localization and SR-released $\mathrm{Ca}^{2+}$ are required. The signal transduction from MTs to mitochondria is mediated at the site of the OMM, where the mitochondria-MT-connecting complex and mitochondrial fusion proteins are located. MT disorganization could regulate mPTP through the OMM complex and $\mathrm{Ca}^{2+}$-sensitive signaling pathway, which also interacts with mitochondrial fusion proteins.

\section{Acknowledgments}

This study was funded by a Japanese Grant-in-Aid from the Japanese Ministry of Education, Culture, Sports, Science, and Technology [23591036 to H.K., 24591045 to H.H.]

\section{Disclosures}

Competing interests: None.

\section{References}

1. Desai A, Mitchison TJ. Microtubule polymerization dynamics. Annu Rev Cell Dev Biol 1997; 13: 83-117.

2. Tsutsui H, Ishihara K, Cooper G 4th. Cytoskeletal role in the contractile dysfunction of hypertrophied myocardium. Science 1993; 260: $682-687$.

3. Zile MR, Green GR, Schuyler GT, Aurigemma GP, Miller DC, Cooper $\mathrm{G} 4$ th. Cardiocyte cytoskeleton in patients with left ventricular pressure overload hypertrophy. J Am Coll Cardiol 2001; 37: 1080-1084.

4. Sato H, Hori M, Kitakaze M, Iwai K, Takashima S, Kurihara H, et al. Reperfusion after brief ischemia disrupts the microtubule network in canine hearts. Circ Res 1993; 72: 361-375.

5. Elrod JW, Molkentin JD. Physiologic functions of cyclophilin D and the mitochondrial permeability transition pore. Circ J 2013; 77: 11111122.

6. Liu X, Hajnoczky G. $\mathrm{Ca}^{2+}$-dependent regulation of mitochondrial dynamics by the Miro-Milton complex. Int J Biochem Cell Biol 2009; 41: $1972-1976$.

7. Mironov SL, Ivannikov MV, Johansson M. $\left[\mathrm{Ca}^{2+}\right]_{i}$ signaling between mitochondria and endoplasmic reticulum in neurons is regulated by microtubules. From mitochondrial permeability transition pore to $\mathrm{Ca}^{2+}$ induced $\mathrm{Ca}^{2+}$ release. J Biol Chem 2005; 280: 715-721.

8. Kidd JF, Pilkington MF, Schell MJ, Fogarty KE, Skepper JN, Taylor $\mathrm{CW}$, et al. Paclitaxel affects cytosolic calcium signals by opening the mitochondrial permeability transition pore. J Biol Chem 2002; 277: $6504-6510$.

9. Kerfant BG, Vassort G, Gomez AM. Microtubule disruption by colchicine reversibly enhances calcium signaling in intact rat cardiac myocytes. Circ Res 2001; 88: E59-E65.

10. Wang X, Schwarz TL. The mechanism of $\mathrm{Ca}^{2+}$-dependent regulation of kinesin-mediated mitochondrial motility. Cell 2009; 136: 163-174.

11. Saotome M, Safiulina D, Szabadkai G, Das S, Fransson A, Aspenstrom $\mathrm{P}$, et al. Bidirectional $\mathrm{Ca}^{2+}$-dependent control of mitochondrial dynamics by the Miro GTPase. Proc Natl Acad Sci USA 2008; 105: 20728 20733.

12. Dorn GW 2nd. Mitochondrial dynamism and cardiac fate: A personal perspective. Circ J 2013; 77: 1370-1379.

13. Papanicolaou KN, Khairallah RJ, Ngoh GA, Chikando A, Luptak I, O'Shea KM, et al. Mitofusin-2 maintains mitochondrial structure and contributes to stress-induced permeability transition in cardiac myocytes. Mol Cell Biol 2011; 31: 1309-1328.

14. Schiff PB, Fant J, Horwitz SB. Promotion of microtubule assembly in vitro by taxol. Nature 1979; 277: 665-667.

15. Schimmel KJ, Richel DJ, van den Brink RB, Guchelaar HJ. Cardiotoxicity of cytotoxic drugs. Cancer Treat Rev 2004; 30: 181-191.

16. Nagasaka S, Katoh H, Niu CF, Matsui S, Urushida T, Satoh H, et al. Protein kinase A catalytic subunit alters cardiac mitochondrial redox state and membrane potential via the formation of reactive oxygen species. Circ J 2007; 71: 429-436.

17. Saotome M, Katoh H, Satoh H, Nagasaka S, Yoshihara S, Terada H, et al. Mitochondrial membrane potential modulates regulation of mi- tochondrial $\mathrm{Ca}^{2+}$ in rat ventricular myocytes. Am J Physiol Heart Circ Physiol 2005; 288: H1820-H1828.

18. Odagiri K, Katoh H, Kawashima H, Tanaka T, Ohtani H, Saotome $\mathrm{M}$, et al. Local control of mitochondrial membrane potential, permeability transition pore and reactive oxygen species by calcium and calmodulin in rat ventricular myocytes. J Mol Cell Cardiol 2009; 46: 989-997.

19. Bers DM. Cardiac excitation-contraction coupling. Nature 2002; 415: 198-205.

20. Zhang K, Heidrich FM, DeGray B, Boehmerle W, Ehrlich BE. Paclitaxel accelerates spontaneous calcium oscillations in cardiomyocytes by interacting with NCS-1 and the InsP3R. J Mol Cell Cardiol 2010; 49: $829-835$.

21. Misko A, Jiang S, Wegorzewska I, Milbrandt J, Baloh RH. Mitofusin 2 is necessary for transport of axonal mitochondria and interacts with the Miro/Milton complex. J Neurosci 2010; 30: 4232-4240.

22. Parra V, Eisner V, Chiong M, Criollo A, Moraga F, Garcia A, et al. Changes in mitochondrial dynamics during ceramide-induced cardiomyocyte early apoptosis. Cardiovasc Res 2008; 77: 387-397.

23. Appaix F, Kuznetsov AV, Usson Y, Kay L, Andrienko T, Olivares $\mathrm{J}$, et al. Possible role of cytoskeleton in intracellular arrangement and regulation of mitochondria. Exp Physiol 2003; 88: 175-190.

24. Rostovtseva TK, Sheldon KL, Hassanzadeh E, Monge C, Saks V, Bezrukov SM, et al. Tubulin binding blocks mitochondrial voltagedependent anion channel and regulates respiration. Proc Natl Acad Sci USA 2008; 105: 18746-18751.

25. Evtodienko YV, Teplova VV, Sidash SS, Ichas F, Mazat JP. Microtubule-active drugs suppress the closure of the permeability transition pore in tumour mitochondria. FEBS Lett 1996; 393: 86-88.

26. Andre N, Braguer D, Brasseur G, Goncalves A, Lemesle-Meunier D, Guise S, et al. Paclitaxel induces release of cytochrome c from mitochondria isolated from human neuroblastoma cells. Cancer Res 2000; 60: $5349-5353$.

27. Howarth FC, Calaghan SC, Boyett MR, White E. Effect of the microtubule polymerizing agent taxol on contraction, $\mathrm{Ca}^{2+}$ transient and L-type $\mathrm{Ca}^{2+}$ current in rat ventricular myocytes. J Physiol 1999; 516 (Pt 2): 409-419.

28. Gao M, Yan X, Weng HR. Inhibition of glycogen synthase kinase 3 beta activity with lithium prevents and attenuates paclitaxel-induced neuropathic pain. Neuroscience 2013; 254: 301-311.

29. Morel M, Authelet M, Dedecker R, Brion JP. Glycogen synthase kinase-3beta and the p 25 activator of cyclin dependent kinase 5 increase pausing of mitochondria in neurons. Neuroscience 2010; 167: $1044-1056$

30. Chen Y, Csordas G, Jowdy C, Schneider TG, Csordas N, Wang W, et al. Mitofusin 2-containing mitochondrial-reticular microdomains direct rapid cardiomyocyte bioenergetic responses via inter-organelle $\mathrm{Ca}^{2+}$ crosstalk. Circ Res 2012; 111: 863-875.

31. de Brito OM, Scorrano L. Mitofusin 2 tethers endoplasmic reticulum to mitochondria. Nature 2008; 456: 605-610.

32. Iwai K, Hori M, Kitabatake A, Kurihara H, Uchida K, Inoue M, et al. Disruption of microtubules as an early sign of irreversible ischemic injury. Immunohistochemical study of in situ canine hearts. Circ Res 1990; 67: 694-706.

33. Gomez AM, Kerfant BG, Vassort G. Microtubule disruption modulates $\mathrm{Ca}^{2+}$ signaling in rat cardiac myocytes. Circ Res 2000; 86: 30-36.

\section{Supplementary Files}

Supplementary File 1

Supplementary methods

Supplementary File 2

Figure S1. Fluorescent imaging of the microtubule network in cardiomyocytes.

Figure S2. Shape of mitochondria in permeabilized myocytes after treatment with paclitaxel or nocodazole.

Figure S3. Effect of paclitaxel on $\mathrm{Ca}^{2+}$ sparks.

Figure S4. Modulation of mitochondrial permeability transition pores (mPTP) by paclitaxel- or nocodazole-mediated ROS production.

Figure S5. Effects of paclitaxel on mitochondrial membrane potential in $\mathrm{H} 9 \mathrm{c} 2$ cells.

Please find supplementary file(s);

http://dx.doi.org/10.1253/circj.CJ-13-1298 